\title{
Research on Optimal Access Point and Capacity of Multiscenario Wind Turbine Based on Voltage Sag
}

\author{
Li Ma, ${ }^{1}$ Chao Zhang ${ }^{1},{ }^{1}$ TaoFeng Liu, ${ }^{2}$ DingRong Tian, ${ }^{1}$ Wei Zhang, ${ }^{1}$ and ShuPing Gao ${ }^{1}$ \\ ${ }^{1}$ College of Electrical and Control Engineering, Xi'an University of Science and Technology, Xi'an 710000, China \\ ${ }^{2}$ State Grid Tongchuan Electric Power Supply Company, Tongchuan, China \\ Correspondence should be addressed to Chao Zhang; 20206227098@stu.xust.edu.cn
}

Received 3 September 2021; Accepted 28 January 2022; Published 28 February 2022

Academic Editor: Ray-Yeng Yang

Copyright $(0) 2022 \mathrm{Li} \mathrm{Ma}$ et al. This is an open access article distributed under the Creative Commons Attribution License, which permits unrestricted use, distribution, and reproduction in any medium, provided the original work is properly cited.

\begin{abstract}
Aiming at reducing the impact on sensitive loads and the economic losses caused by voltage sags in the distribution network containing wind turbines, a multiobjective mathematical model of the optimal access point and access capacity is established from the three perspectives of the basic costs of wind turbines, the economic losses of voltage sags, and the economic losses of the power grid. A scene generation method based on the similarity of wind curve changes and a scene reduction algorithm based on DPC \& $k$-means are proposed to solve the mathematical problem of wind turbine uncertainty. Based on the analysis of the voltage sag of the distribution network containing wind turbines, the evaluation indicator of the voltage sag depth of the bus and the evaluation indicator of the economic loss of the voltage sag of the sensitive load are proposed. The simulation results of the IEEE 33-bus system show that the reasonable access of wind turbines can help reduce the power loss and voltage sag of the distribution network, thereby ensuring the safe and economical operation of the system.
\end{abstract}

\section{Introduction}

Wind turbine has the characteristics of low pollution and strong sustainability compared with traditional thermal power generation. The proportion of wind turbine in the distribution network is gradually increasing. Taking into account the intermittency and uncertainty of wind turbine output and the impact on the distribution network, the selection of the access point and capacity of wind turbines has become one of the research focuses. Most power quality problems are caused by voltage sags, which would make sensitive loads unable to operate normally and cause economic losses. The access of wind turbines has a mitigation effect on voltage sag, so, from the perspective of alleviating voltage sags, the selection of the wind turbine optimal access plan is of great significance.

Regarding the problem of access point and capacity of wind turbines, most researchers solve it from three aspects as follows.

(1) The model of wind turbines: most of the articles use wind turbines as a power source with constant output for modelling. For actual wind turbines, due to the influence of uncertain factors such as wind speed, there are uncertainties in the output of the wind turbine. In order to solve such problems more accurately, the authors in [1] proposed a new voltage stability model based on voltage, wind energy permeability, short-circuit capacity, and short-circuit impedance ratio $(X / R)$, so that engineers can predict the voltage changes at the public access point of wind turbines. The authors in [2] use multiscenario probability models to analyze the time sequence of DG output and load demand. The authors in [3] proposed a new measurement method of wind power change rate and studied the influence of wind power change rate on the access capacity of independent wind power system.

(2) Optimization goal: in [4], the loss sensitivity factor (LSF) is used to determine the installation position of DG with the objective of reducing the bus loss of 
radial distribution network. Intelligent water drop (IWD) algorithm is used to determine the installed capacity of DG. In [5], the optimal capacity of DG is determined by meeting the maximum allowable line capacity limit (MAlCl). The authors in [6] use weighted voltage support capability index (WVSAI) based on sensitivity analysis to quantify the impact of PV-STATCOM on voltage recovery efficiency under voltage sag. The authors in [7] proposed a multienergy power supply multipoint layout planning model based on complex adaptive system theory. The authors in [8] proposed a three-objective optimization planning model for wind turbines and capacitors considering the uncertainty of plug-in vehicle load demand, wind speed, and load. The specific optimization targets are voltage stability indicators, total costs, and greenhouse gas emissions.

(3) Optimization algorithm: The authors in [9] proposed a computer intelligent algorithm based on evolutionary programming (EP) to solve the problem of DG access point and capacity. Among them, the goal is to minimize the power loss of the system and to ensure the voltage status of the entire network bus. The authors in [10] use particle swarm optimization algorithm (PSO) to solve the problem. For the purpose of power system economy, the authors in [11] proposed an optical optimization algorithm (OIO) to improve the current problem. In [12], an improved long-horned beetle search algorithm is used to solve the problem of distribution network planning with DG. Among them, the improvement of the algorithm is based on the Monte Carlo rule in the simulated annealing algorithm. In [13], a fuzzy genetic algorithm (FGA) is proposed, which can maximize the economic benefits of the project.

It can be known that the consideration of wind speed uncertainty in the abovementioned references is only limited to fixed scenarios; the uncertainty of wind speed is not described in terms of actual conditions. In addition, in the actual problems of selection of access point and capacity of wind turbines, most of them determine the optimal access point and capacity from an economic point of view. The influence of wind turbine access on the reliability of the distribution network is not considered.

Wind power is uncertain and difficult to be expressed with actual mathematical models. Multiscenario analysis method is used to simulate the uncertainty of wind power in this paper. Scenes are generated based on the characteristics of wind curve change similarity, and a scene reduction algorithm based on DPC and $k$-means is used to delete the scene reasonably. In order to evaluate the mitigation degree of wind turbines access to voltage sag caused by fault, an evaluation indicator for the voltage sag depth is proposed. A DG multiobjective optimization model is established using the indicator of voltage sag depth. This model considers the impact of wind turbine access on the reliability of the distribution network while considering economy. An elite sampling algorithm suitable for engineering is used to solve the model, and example simulation was carried out in the IEEE 33-bus system to verify the feasibility and effectiveness of the method.

The main contributions of this paper are as follows:

(1) This paper proposes a scene reduction method based on the combination of DPC and $K$-means algorithm. This method reduces the number of scenes according to the demand, thereby simplifying the complexity of calculations.

(2) A multiobjective mathematical model for the optimization of wind turbine access point and capacity selection is established. This model considers the economic loss of voltage sag while considering the operation and maintenance costs of wind turbines and the economic loss of power grid loss. Combining the research methods in this paper can solve the problems related to voltage sag and economy in the distribution network containing wind turbine.

(3) This paper proposes the evaluation indicator of the voltage sag depth of the bus. This indicator is used to calculate the economic loss of sensitive loads caused by voltage sag and to determine the access point and capacity of the wind turbine.

This paper is organized as follows. Section 2 presents a wind power multiscenario analysis method based on wind speed curve change similarity. Section 3 describes the optimization of multitarget capacity and location selection of wind turbine. In Section 4, elite sampling algorithm is used to solve the mathematical model. In Section 5, a simulation based on IEEE 33-bus system is presented and an analysis of the results is also described in Section 5. At last, conclusions are discussed in Section 6.

Nomenclature list is shown in Table 1.

\section{Wind Power Multiscenario Analysis Method Based on Wind Speed Curve Change Similarity}

Due to the influence of the random change of wind speed, the output of wind turbine is uncertain. Therefore, in the mathematical model of optimal access point and capacity of wind turbine, if the output of generator is regarded as a constant value, there may be a large error between the final calculation result and the actual result, which cannot simulate the real output of wind turbine. Therefore, it is necessary to take the time sequence characteristics of wind power output into consideration when selecting optimal access point and capacity of wind turbines.

The core content of multiscenario analysis method is to change the uncertain factors into many easily obtained and calculated deterministic factors. The multiscenario analysis method has high adaptability to random problem. Therefore, this method is used to simulate the uncertainty of wind power output. 
TABle 1: Nomenclature list.

\begin{tabular}{lc}
\hline $\mathrm{CS}$ & Trend similarity between two curves \\
$w(s)$ & The probability of occurrence of each scene $s$ \\
$\operatorname{Pr}(s)$ & The normalized probability for each scene \\
$\mathrm{CH}(+)$ & Ratio of within-class deviation to between-class deviation \\
$\operatorname{Tr}\left(S_{B}\right)$ & Trace of the between-class deviation matrix \\
$\operatorname{Tr}\left(S_{w}\right)$ & Trace of the within-class deviation matrix \\
$U^{(x)}$ & Voltage matrix when $x$-type short-circuit fault occurs \\
$C^{(x)}$ & Voltage major axis matrix when $x$-type short-circuit fault occurs \\
$P^{(x)}$ & Voltage minor axis matrix when $x$-type short-circuit fault occurs \\
$M^{(x)}$ & Voltage sag matrix when $x$-type short-circuit fault occurs \\
$R^{(x)}$ & Voltage sag depth matrix when $x$-type short-circuit fault occurs \\
$\lambda_{x}$ & Failure probability of $x$-type short-circuit fault \\
$n$ & The total number of buses \\
$F_{j}^{(x)}$ & The depth indicator when $x$-type short-circuit fault occurs \\
$F_{j}$ & The average depth of voltage sag \\
$f_{\text {sen }}$ & The total economic loss of sensitive load caused by voltage sag \\
$f_{1}$ & The total cost of wind turbine accessing \\
$f_{i, r m}$ & The annual operation and maintenance cost of the wind turbine accessed to the node $i$ \\
$f_{i, \text { fault }}$ & The failure cost of the wind turbine accessed to the node $i$ \\
$P_{\text {loss }}$ & The network loss \\
$f_{2}$ & The total economic cost caused by network loss after considering multiscene \\
\hline
\end{tabular}

\subsection{Scene Generation Based on Similarity of Wind Speed Curve} Change. The traditional scene generation method classifies the wind speed or wind turbine output according to four seasons, but the wind speed data of different regions in the world extracted by HOMER shows that the wind speed will also change with the location and the wind speed changes in different regions in four seasons are not the same. The fixed mode of wind speed division cannot guarantee the correctness of the subsequent mathematical model; the traditional scheme of mathematical model has low adaptive ability. In order to better adapt to the characteristics of wind speed in different regions, this paper proposes a scene generation process based on the similarity of wind speed curve change trend on the basis of [14]. Comparing the daily wind speed changes in 365 days, those with similar wind speed change curves are regarded as one kind. When the number of wind speed curves of a certain type is small, the probability of this occurrence is considered small, and it is deleted from the original database. Through the above classification process, the process of scene generation is further optimized, and the accuracy of subsequent calculation is improved.

In this paper, the absolute error of enclosing area between different wind speed curves is used to describe the trend similarity of curves [15]. When the trend similarity of two curves is smaller, the change trend of the two curves is closer. When the two curves coincide, the curve trend similarity is 0 .

If there are two wind speed curves $w 1(t)$ and $w 2(t)$, the trend similarity of the two curves is CS, and the specific calculation formula is as follows.

$$
\mathrm{CS}=\frac{\sum_{t=0}^{23}|w 1(t)+w 1(t+1)-w 2(t)-w 2(t+1)|}{\sum_{t=0}^{23}|w 2(t)+w 2(t+1)|} .
$$

The optimized scene generation process is as follows.
Step 1: combined with the geographical and meteorological data, the wind speed changes of a certain area in each period of the whole day in a year are obtained through the HOMER.

Step 2: calculate the trend similarity between curves according to formula (1) and classify the wind speed according to the principle of curve trend similarity.

Step 3: assume that the error $\Delta \mathrm{D}$ between the actual data and the typical data of each period obeys the corresponding probability distribution function (PDF). The wind speed data that have been classified according to the trend similarity principle are extracted. The probability density function $\operatorname{PDF}(t=1,2, \ldots, 23,24)$ is calculated by the error between the typical output value and the actual value of each type of wind speed curve. The random variable expression corresponding to each scene can be expressed by the following formulas:

$$
\begin{aligned}
\mathrm{w} v_{x, t, s} & =w \mathrm{v}_{x, t}^{o}+\Delta w v_{x, t, s}, \\
L_{x, t, s} & =L_{x, t}^{o}+\Delta L_{x, t, s},
\end{aligned}
$$

where $\mathrm{wv}_{x, t, s}$ is the $x$-th wind speed actual value in scene $s$ at moment $t . \mathrm{wv}_{x, t}^{\mathrm{o}}$ is the $x$-th wind speed typical value in scene $s$ at moment $t . \Delta \mathrm{wv}_{x, t, s}$ is the difference between the $x$-th actual value and the $x$-th typical value in scene $s$ at moment $t . L_{x, t, s}$ is the $x$-th load actual value in scene $s$ at moment $t . L_{x, t}^{o}$ is the $x$-th load typical value in scene $s$ at moment $t . \Delta L_{x, t, s}$ is the difference between the $x$-th actual value and the $x$-th typical value in scene $s$ at moment $t$.

Step 4: divide the PDF generated by Step 3 into 7 equal parts, and the schematic diagram is shown as follows.

In Figure 1, $\alpha_{i, t}$ represents the probability of occurrence in the error interval of the wind speed $i$ at moment $t$. 


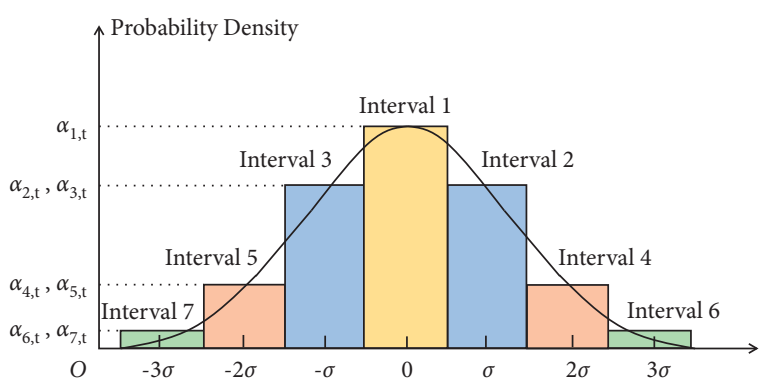

FIgURE 1: Discrete probability distribution function of wind speed error.

Step 5: in order to facilitate the selection of scene, the interval in Step 4 is probability standardized.

Step 6: use Monte Carlo sampling/roulette selection method [16] to obtain the scene $s$.

$$
\begin{aligned}
& s=\left\{W_{1, t, s}^{x}, \ldots, W_{7, t, s}^{x}\right\}, \\
& t=1,2, \ldots, 24 .
\end{aligned}
$$

The scene $s$ is a parameter consisting of 0 and $1 . W_{i, t, s}^{x}$, respectively, represents the selection of the error interval of the wind speed $i$ in the scene $s$ at the moment $t$.

Step 7: calculate the probability of scene $s$ and standardize it.

$$
\omega(s)=\prod_{t=1}^{24}\left[\sum_{i=1}^{7}\left(W_{i, t, s}^{x} \alpha_{i, t}\right)\right] .
$$

where $w(s)$ is the occurrence probability of each scene $s$.

$$
P_{r}(s)=\frac{\omega(s)}{\sum_{s=1}^{N S} \omega(s)} .
$$

where $P_{r}(s)$ is the normalized probability for each scene.

\subsection{Scene Reduction Based on DPC and K-Means.} Generally, the number of the divided scenes should be large enough to meet the requirements of calculation accuracy, but the large number of scenes will inevitably increase the calculation complexity of the model. In order to solve the contradiction between calculation accuracy and calculation complexity, it is necessary to limit the number of scenes.

Clustering by fast search and finding density peaks (DPC) [17] is a new clustering algorithm proposed by Alex and Alessandro in 2014. This algorithm can find the density peaks of arbitrary shape data sets, select the number of clusters according to the decision graph, and has an efficient sample point allocation strategy [18]. The advantage of this method is that it can select the cluster center of discrete points better, but the disadvantage is that it takes a long time to complete the whole process of clustering. $K$-means clustering algorithm is a traditional clustering algorithm based on iteration. It is widely used because of its simple principle, easiness to write algorithm, and good clustering effect. The main parameters of $K$-means algorithm are the number of initial clustering centers and the location of the centers. The performance of $K$-means clustering depends on the selection of the above parameters. By comparing the pros and cons of the two methods, this paper proposes a method based on the combination of DPC algorithm and $K$-means algorithm to optimize the clustering process.

Step 1: import the $N_{s}$ original scene data into the DPC algorithm to obtain the initial cluster center point set, namely, the preliminary cluster center scene set.

Step 2: import the initial cluster center scene set obtained in the first step into the $k$-means algorithm as the parameters for the first iteration. Finally, through the clustering algorithm based on the combination of DPC $\& K$-means, we can get the central scene set and the number of scenes after clustering.

Step 3: assign the original scene data to the corresponding cluster center field according to the distance between the original scene data and the cluster center field and label the original scene by category, so as to complete the first clustering. The probability of the scene represented by the cluster center is calculated by the cumulative probability of all such scenes.

Step 4: considering the characteristics of clustering center in $k$-means algorithm, it is necessary to iterate the clustering center for many times. Therefore, Step 2 and Step 3 are repeated until the final result meets the accuracy requirements or reaches the maximum number of iterations.

The corresponding cluster centers are obtained through the above algorithm, but the quality of the results cannot be intuitively evaluated. In order to reflect the pros and cons of the selected clustering centers, this paper uses clustering validity indicator $\mathrm{CH}^{(+)}$[19] to determine the results of the scene reduction algorithm, so as to further improve the accuracy of the scene reduction algorithm. The cluster validity indicator $\mathrm{CH}^{(+)}$is the ratio of within cluster deviation to intercluster deviation, and its expression is shown in the following formula:

$$
\mathrm{CH}^{(+)}=\frac{\operatorname{Tr}\left(S_{B}\right) /(K-1)}{\operatorname{Tr}\left(S_{w}\right) /(N-K)}=\frac{N-K}{K-1} \frac{\operatorname{Tr}\left(S_{B}\right)}{\operatorname{Tr}\left(S_{w}\right)} .
$$

In formula (7), $N$ represents the number of original data, $K$ represents the number of clusters, $\operatorname{Tr}\left(S_{B}\right)$ represents the trace of interclass deviation matrix, and $\operatorname{Tr}\left(S_{w}\right)$ represents the trace of intraclass deviation matrix. The calculation of $\operatorname{Tr}\left(S_{B}\right)$ and $\operatorname{Tr}\left(S_{w}\right)$ is shown in the following formulas:

$$
\operatorname{Tr}\left(S_{B}\right)=\sum_{i=1}^{K} n_{i} \times d\left(v_{j}, \bar{v}\right) .
$$

In formula (8), $n_{i}$ represents the number of data $i$ and $v_{j}$ represents the center point of data $j . \bar{v}$ represents the central point of the entire data set; $\mathrm{d}\left(v_{j}, \bar{v}\right)$ is the distance between $v_{j}$ and $\bar{v}$. 


$$
\operatorname{Tr}\left(S_{w}\right)=\sum_{i=1}^{K} \sum_{j=1}^{n_{i}} d\left(x_{j}, v_{i}\right) .
$$

In formula (9), $x_{j}$ represents the data $j$ of the class $I$ and $v_{i}$ represents the center point of data $i . d\left(x_{j}, v_{i}\right)$ is the distance between $x_{j}$ and $v_{i}$.

Through the analysis of the above indicators, it can be seen that the denominator of this indicator measures the tightness within the class and the numerator measures the separation between classes. Therefore, it can be known that when the indicator $\mathrm{CH}^{(+)}$is larger, the data of the same classes are closer and the data of different classes are more scattered; that is, the clustering effect of the algorithm is better. When the number of clusters is $x$, its indicator $\mathrm{CH}^{(+)}$ is the largest, which can prove that $x$ is the best number of clusters.

\section{The Optimization of Multitarget Optimal Access Point and Capacity of Wind Turbine}

3.1. Analysis and Evaluation of Voltage Sag Based on Voltage Ellipse Parameters. The Clarke transformation was firstly proposed by Edith Clarke in 1951 [20]. The three-phase voltage can form space rotation vector $\vec{E}(t)$ through Clarke transformation. The space rotation vector can be expressed as shown in

$$
\vec{E}(t)=\frac{2}{3}\left[\begin{array}{lll}
1 & e^{j 2 \pi / 3} & e^{j 4 \pi / 3}
\end{array}\right]\left[\begin{array}{c}
v_{a}(t) \\
v_{b}(t) \\
v_{c}(t)
\end{array}\right]=E^{+} e^{j \omega t}+E^{-} e^{-j \omega t} .
$$

Among them, $E^{+}=\left|E^{+}\right| e^{j \psi^{+}} E^{-}=\left|E^{-}\right| e^{j \psi^{-}}$. If the parameter $c$ is defined as major axis and the parameter $p$ is minor axis, the formula can be defined as follows.

$$
\begin{aligned}
& c=\left\|E^{+}|+| E^{-}\right\|, \\
& p=\left\|E^{+}|-| E^{-}\right\| .
\end{aligned}
$$

According to the above analysis, the corresponding voltage ellipse parameters can be obtained by plotting the space rotation vector $\vec{E}(t)$ in a complex plane. The voltage sag caused by different fault types can be expressed by corresponding voltage ellipse parameters [21, 22]. Therefore, voltage ellipse parameters can be used to classify and characterize voltage sag.

Compared with the voltage sag caused by line fault, the voltage sag caused by bus fault will have a greater adverse impact on the distribution network. Therefore, this paper mainly discusses the impact of bus fault on the distribution network. Assuming that each bus in the distribution network has four types of short-circuit faults (all metallic shortcircuit faults), the voltage value of each bus is calculated by the method of fault point [23] and the voltage matrix is established under the given grid structure (where $x=1,2,3$, and 4 represent single-phase grounding, two-phase short- circuit, two-phase grounding, and three-phase short-circuit, respectively).

$$
U^{(x)}=\left[\begin{array}{cccccc}
u_{11}^{(x)} & u_{12}^{(x)} & \ldots & u_{1 j}^{(x)} & \ldots & u_{1 n}^{(x)} \\
u_{21}^{(x)} & u_{22}^{(x)} & \ldots & u_{2 j}^{(x)} & \ldots & u_{2 n}^{(x)} \\
\vdots & \vdots & \ddots & & & \vdots \\
u_{i 1}^{(x)} & u_{i 2}^{(x)} & & u_{i j}^{(x)} & & u_{i n}^{(x)} \\
\vdots & \vdots & & & \ddots & \vdots \\
u_{n 1}^{(x)} & u_{n 2}^{(x)} & \ldots & u_{n j}^{(x)} & \ldots & u_{n n}^{(x)}
\end{array}\right] .
$$

$U^{(x)}$ in formula (12) is the voltage matrix under shortcircuit type $x$, which $u_{i j}^{(x)}$ represents the three-phase voltage on bus $j$ when fault type $x$ occurs on bus $i$. Clarke transform is applied to the three-phase voltage of each bus to extract the major axis parameters and minor axis parameters of the corresponding bus.

$$
\begin{aligned}
P^{(x)} & =\left[\begin{array}{cccccc}
p_{11}^{(x)} & p_{12}^{(x)} & \ldots & p_{1 j}^{(x)} & \ldots & p_{1 n}^{(x)} \\
p_{21}^{(x)} & p_{22}^{(x)} & \ldots & p_{2 j}^{(x)} & \ldots & p_{2 n}^{(x)} \\
\vdots & \vdots & \ddots & & & \vdots \\
p_{i 1}^{(x)} & p_{i 2}^{(x)} & & p_{i j}^{(x)} & & p_{i n}^{(x)} \\
\vdots & \vdots & & & \ddots & \vdots \\
p_{n 1}^{(x)} & p_{n 2}^{(x)} & \ldots & p_{n j}^{(x)} & \ldots & p_{n n}^{(x)}
\end{array}\right], \\
C^{(x)} & =\left[\begin{array}{cccccc}
c_{11}^{(x)} & c_{12}^{(x)} & \ldots & c_{1 j}^{(x)} & \ldots & c_{1 n}^{(x)} \\
c_{21}^{(x)} & c_{22}^{(x)} & \ldots & c_{2 j}^{(x)} & \ldots & c_{2 n}^{(x)} \\
\vdots & \vdots & \ddots & & & \vdots \\
c_{i 1}^{(x)} & c_{i 2}^{(x)} & & c_{i j}^{(x)} & & c_{i n}^{(x)} \\
\vdots & \vdots & & & \ddots & \vdots \\
c_{n 1}^{(x)} & c_{n 2}^{(x)} & \ldots & c_{n j}^{(x)} & \ldots & c_{n n}^{(x)}
\end{array}\right] .
\end{aligned}
$$

The $P^{(x)}$ and $C^{(x)}$ in formulas (13) and (14) represent the voltage major axis matrix and the voltage minor axis matrix under the short-circuit type $x$, respectively. The $p_{i j}^{(x)}$ and $c_{i j}^{(x)}$ represent the major axis parameters and minor axis parameters of the bus $j$ when the bus $i$ has short-circuit fault type $x$.

According to the voltage ellipse parameters, the specific rules for judging whether there is voltage sag on the bus are as follows [21]:

(1) When the major voltage axis parameters $p_{i j}^{(x)}$ and minor voltage axis parameters $c_{i j}^{(x)}$ of the bus are in the range [0.9333,1.06667], the bus is normal; that is, there is no voltage sag.

(2) When the major axis parameters $p_{i j}^{(x)}$ and minor axis parameters $c_{i j}^{(x)}$ of the bus are not in the range of $[0.9333,1.06667]$, and the minor axis parameters $c_{i j}^{(x)}$ are less than 0.9333 , the voltage sag occurs on the bus. Through the above analysis, the voltage sag matrix with short-circuit type $x$ is obtained. 


$$
M^{(x)}=\left[\begin{array}{cccccc}
m_{11}^{(x)} & m_{12}^{(x)} & \ldots & m_{1 j}^{(x)} & \ldots & m_{1 n}^{(x)} \\
m_{21}^{(x)} & m_{22}^{(x)} & \ldots & m_{2 j}^{(x)} & \ldots & m_{2 n}^{(x)} \\
\vdots & \vdots & \ddots & & & \vdots \\
m_{i 1}^{(x)} & m_{i 2}^{(x)} & & m_{i j}^{(x)} & & m_{i n}^{(x)} \\
\vdots & \vdots & & & \ddots & \vdots \\
m_{n 1}^{(x)} & m_{n 2}^{(x)} & \ldots & m_{n j}^{(x)} & \ldots & m_{n n}^{(x)}
\end{array}\right]
$$

The $M^{(x)}$ in formula (15) is the voltage sag matrix under short-circuit type $x . m_{i j}^{(x)}$ represents the situation of bus $j$ when short-circuit faults type $x$ occurs on bus $i$. If voltage sag occurs on the bus $j$, let $m_{i j}^{(x)}=1$. On the contrary, if there is no voltage sag on the bus $j$, let $m_{i j}^{(x)}=0$.

The voltage sag depth matrix $R^{(x)}$ can be obtained through the voltage matrix $U^{(x)}$ and the voltage sag matrix $M^{(x)}$, the formula can be defined as follows.

$$
\begin{aligned}
r_{i j}^{(x)}= & \left(1-u_{i j, \min }^{(x)}\right) m_{i j}^{(x)}, \\
R^{(x)}= & {\left[\begin{array}{cccccc}
r_{11}^{(x)} & r_{12}^{(x)} & \ldots & r_{1 j}^{(x)} & \ldots & r_{1 n}^{(x)} \\
r_{21}^{(x)} & r_{22}^{(x)} & \ldots & r_{2 j}^{(x)} & \ldots & r_{2 n}^{(x)} \\
\vdots & \vdots & \ddots & & & \vdots \\
r_{i 1}^{(x)} & r_{i 2}^{(x)} & & r_{i j}^{(x)} & & r_{i n}^{(x)} \\
\vdots & \vdots & & & \ddots & \vdots \\
r_{n 1}^{(x)} & r_{n 2}^{(x)} & \ldots & r_{n j}^{(x)} & \ldots & r_{n n}^{(x)}
\end{array}\right] }
\end{aligned}
$$

In formula (16), $u_{i j \text {,min }}^{(x)}$ represents the phase voltage with the smallest voltage value on bus $j$ when an $x$-type fault occurs on bus $i$.

3.2. Analysis and Evaluation of Economic Loss Caused by Voltage Sag of Sensitive Load. From the column $j$ of the voltage sag depth matrix $R^{(x)}$, the occurrence of voltage sags on bus $j$ when other bus failures occur can be obtained. Therefore, from the column information, the depth indicator $F_{j}^{(x)}$ of the voltage sag caused by the fault type $m$ on the bus $j$ by other buses can be obtained; see the following formula for details:

$$
F_{j}^{(x)}=\sum_{i=1}^{N} r_{i j}^{(x)} .
$$

Consider the average depth $F_{j}$ of voltage sag when four short-circuit faults occur on the bus $j$ in turn. See the following formula for details:

$$
F_{j}=\sum_{x=1}^{4} \lambda_{x} F_{j}^{(x)}
$$

In order to measure the economic loss of sensitive load caused by voltage sag, this paper proposes the economic loss indicator of sensitive load based on depth indicators $F_{j}^{(x)}$ and $F_{j}$.

$$
f_{\text {sen }}=\sum_{j=1}^{\chi} \tau_{j} F_{j}
$$

$f_{\text {sen }}$ is the total economic loss of sensitive load caused by voltage sag. $\tau_{j}$ is the average economic loss of sensitive load on node $j$ caused by a single voltage sag event. $\chi$ is the total number of nodes accessed to sensitive loads.

In order to further reflect the impact of wind turbine access on the economic loss of sensitive load voltage sag, the influence of wind power multiscenario is considered in formula (20), and then the calculation formula of sensitive load voltage sag economic loss is changed to the following formula:

$$
f_{\text {sen }}=\sum_{m=1}^{\mathrm{NM}}\left\{d_{w, m} \sum_{s=1}^{\mathrm{NS}_{m}}\left[P_{r w}(s) \sum_{t=1}^{24} f_{\mathrm{sen}, m, s, t}\right]\right\} .
$$

In formula (21), $f_{\text {sen, } m, s, t}$ is the economic loss of sensitive load voltage sag in scene $s$ at moment $t$ corresponding to the wind speed type $m$ considering the time sequence of wind turbine output.

3.3. Objective Function. The objective function in this paper includes the basic cost of wind turbine, the economic loss of voltage sag, and the economic loss of power grid.

3.3.1. Basic Cost of Wind Turbine. The basic cost of wind turbine is mainly composed of three parts. One is the onetime investment and installation cost of wind turbine. The second is the operation and maintenance cost of wind turbine in the later stage. The third is the cost of failure cost. The establishment of the third part is to consider the cost incurring when the wind turbine is not put into the distribution network and the actual wind speed is lower than the cut-in wind speed of the wind turbine.

$$
\begin{aligned}
\mathrm{f}_{1} & =\sum_{\mathrm{i}=1}^{\mathrm{N}_{\mathrm{DG}}}\left(f_{i, r m}+f_{i, i n v}\right), \\
f_{r m} & =\sum_{m=1}^{\mathrm{NM}}\left\{d_{w, m} \sum_{s=1}^{\mathrm{NS}_{m}}\left[P_{r w}(s) \sum_{t=1}^{24} f_{m, s, t, r m}\right\},\right. \\
f_{\text {fault }} & =\sum_{m=1}^{\mathrm{NM}}\left\{d_{w, m} \sum_{s=1}^{\mathrm{NS}_{m}}\left[P_{r w}(s) \sum_{t=1}^{24} f_{m, s, t, \text { fault }}\right]\right\} .
\end{aligned}
$$

In formula (22), $f_{1}$ is the total cost of wind turbine accessing. $f_{i, r m}$ is the annual operation and maintenance cost of the wind turbine accessed to the node $i$, and the specific calculation formula is shown in formula (23). $f_{i \text {,inv }}$ is the one-time investment and installation cost of the wind turbine accessed to the node $i . f_{i \text {,fault }}$ is the failure cost of the wind turbine accessed to the node $i$, and the specific calculation formula is shown in (24). $N_{\mathrm{DG}}$ is the total number of wind turbines accessed to the distribution network. NM is the total number of wind speed categories; $\mathrm{NS}_{m}$ is the total number of scenes under wind speed type $m ; d_{w, m}$ is the 
number of days under wind speed type $m . f_{m, s, t, r m}$ is the operation and maintenance cost of the wind turbine under the scene $s$ of the wind speed type $m$ at moment $t$. Considering that when the wind speed is lower than the cut-in wind speed, the wind turbine will exit the distribution network. At this time, the wind turbine cannot be used as a power source to supply power to the distribution network. Therefore, let $f_{m, s, t \text {,fault }}$ be the failure cost of the wind turbine under the scene $s$ of the wind speed type $m$ at moment $t$. $P_{r \mathrm{w}}(s)$ is the normalized probability for wind speed scene $s$.

3.3.2. Economic Loss of Distribution Network. Under a certain access scheme, the economic loss of power grid loss can be calculated by the following formulas:

$$
P_{\text {loss }}=\sum_{i=1}^{N} \sum_{j=1}^{N}\left[U_{i}^{2} Y_{i j} \cos \theta_{i j}-U_{i} U_{j} Y_{i j} \cos \left(\delta_{j i}+\theta_{i j}\right)\right] \text {, }
$$

where $P_{\text {loss }}$ is the network loss, $U_{i}, U_{j}$ are the voltage of node $i$ and $j, Y_{i j}$ is the admittance of branch $i-j, \theta_{i j}$ is the impedance angle of branch $i-j$, and $\delta_{j i}$ is the phase angle difference of node voltage.

$$
f_{2}=\delta P_{\text {loss }}
$$

Among them, $f_{2}$ is the economic cost caused by network loss and $\delta$ is the economic cost caused by unit network loss.

After considering the influence of scene on network loss, the corresponding economic loss formula of network loss is calculated by the following formulas:

$$
\begin{aligned}
f_{2} & =\sum_{m=1}^{\mathrm{NM}}\left\{d_{w, m} \sum_{s=1}^{\mathrm{NS}_{m}}\left[P_{r w}(s) \sum_{t=1}^{24} f_{2, m, s, t}\right]\right\} . \\
f_{2, m, s, t} & =\sum_{i=1}^{\mathrm{LN}}\left\{d_{l, i} \sum_{c=1}^{\mathrm{LC}}\left[P_{L}(c) \sum_{t=1}^{24} f_{2, m, s, i, c, t}\right\} .\right.
\end{aligned}
$$

In formula (27), $f_{2}$ is the total economic cost caused by network loss after considering multiscene. Among them, LN is the total number of load curve categories and $\mathrm{LC}_{i}$ is the total number of scenes under the load type $m ; d_{m}$ is the number of days under the wind speed type $m . f_{2, m, s, t}$ is the economic loss of network loss at the moment $t$ in the scene $s$ corresponding to the wind speed type $m$. In formula (28), $f_{2, m, s, i, c, t}$ is the economic loss of network loss at moment $t$ in scene $s$ corresponding to wind speed type $m$ and scene $c$ corresponding to load type $i$. $P_{L}(c)$ is the standardized probability for load scene $c . d_{l, m}$ is the number of days under the load $m$.

3.3.3. Economic Loss of Sensitive Load Caused by Voltage Sag. Under a certain access scheme, after considering the influence of multiscenes, the calculation formula of voltage sag economic loss of sensitive load is as follows:

$$
f_{\mathrm{sen}}=\sum_{m=1}^{\mathrm{NM}}\left\{d_{w, m} \sum_{s=1}^{\mathrm{NS}_{m}}\left[P_{r w}(s) \sum_{t=1}^{24} f_{\mathrm{sen}, m, s, t}\right]\right\} .
$$

In formula (29), $f_{\text {sen, } m, s, t}$ is the economic loss of sensitive load voltage sag at the moment $t$ corresponding to the scene $s$ of the wind speed type $m$.

\subsection{Constraint Condition}

(1) Voltage variable inequality constraints: All node voltages must meet the

$$
U_{i \min } \leq U \leq U_{i \max }
$$

In formula (30), $U_{i \text { min }}$ is the lower limit of allowable voltage value of node $i . U_{i \max }$ is the upper limit of allowable voltage value of node $i$.

(2) Branch current constraint:

$$
\left|P_{i j}\right| \leq P_{i j, \max }
$$

In formula (31), $P_{i j}$ and $P_{i j \text {, max }}$ are, respectively, transmission power and the maximum allowed transmission power between node $i$ and node $j$.

(3) The total capacity constraint of the wind turbine installed in the system is as follows:

$$
\sum_{i=1}^{N_{\mathrm{DG}}} S_{i} \leq \eta S
$$

Generally, the total installed capacity of wind turbines in distribution network should not exceed $10 \% \sim 25 \%$ of the maximum capacity of power grid. Therefore, in formula (32), $S_{i}$ is the rated active power of the wind turbine accessed to the node $i . \eta$ is the capacity penetration of distributed generation. $S$ is the total rated active power of the load accessed to the distribution network.

(4) The rated capacity constraints of wind turbine accessed to single node are as follows:

$$
0 \leq P_{i} \leq P_{i, \max }
$$

In formula (33), $P_{i}$ is the rated active power of the wind turbine accessed to the node $i . P_{i, \max }$ is the maximum rated active power of the wind turbine that the node $i$ is allowed to access.

The mathematical model of capacity and location selection of wind turbine optimization problem is to minimize the basic cost, network loss, and voltage sag economic loss of wind turbine under the above constraints.

$$
\begin{array}{r}
\min f=\left[f_{1}, f_{2}, f_{\text {sen }}\right]^{T}, \\
g(x) \leq 0, \\
h(x)=0 .
\end{array}
$$

Among them, $\left[f_{1}, f_{2}, f_{\text {sen }}\right]$ is the objective function of basic cost, economic cost of network loss, and economic loss of voltage sag. $g(x)$ and $h(x)$ are inequality constrained function and equality constrained function, respectively. 


\section{Elite Sampling Algorithm}

In this paper, elite sampling algorithm is used to solve the problem of optimal access point and capacity of wind turbine. Its main features are simplicity and easiness to understand, small amount of calculation, and high applicability [24]. So, it can be better used in optimization problems. In the elite sampling algorithm, when the number of solutions is different, the specific steps of the algorithm and the relevant parameters in the sampling process will also change. In this paper, the number of solutions is limited, so we only introduce the elite sampling algorithm when the number of solutions is finite.

4.1. The Basic Principle of Elite Sampling Algorithm. Assuming that the total number of individuals in the whole solution space is $M$, all the solutions in the whole solution space are sorted by performance and then assume that the first $n$ solution in the performance ranking is the elite solution. Therefore, after generating $N$ solutions randomly, the probability of not being in the first $n$ solution is $(1-n / M)(1-n / M-1) \ldots[1-n / M-(N-1)]$.

Suppose that the probability that none of the randomly generated $N$ candidate solutions are in the first $n$ is not greater than $q \%$; then there is

$$
\left(1-\frac{n}{M}\right)\left(1-\frac{n}{M-1}\right) \ldots\left[1-\frac{n}{M-(N-1)}\right] \leq q \% \text {. }
$$

On the basis of formula (35), the minimum number $N_{\text {min }}^{\prime}$ of samples needed for the problem can be obtained by iterative calculation. As long as the number of randomly generated candidate solutions is not less than $N_{\min }^{\prime}$, there is a possibility of $(1-q \%)$ among the $N_{\min }^{\prime}$ candidate solutions, at least one of which is the first $n$ satisfactory solution.

The specific steps are as follows:

Step 1: initialize parameter $M$ and $n, N=1, t=1$

Step 2: calculate $t=t(1-n / M+1-N)$

Step 3: judge whether $t \leq q \%$ is true? If yes, continue with Step 4; otherwise, $N=N+1$; return to Step 2 .

Step 4: $N_{\min }^{\prime}=N$

In order to improve its efficiency, parallel algorithm can be used in the process of analyzing and evaluating each candidate solution. In actual engineering practice, it is usually enough to obtain elite solutions with better effects and economic efficiency for solving practical problems.

4.2. Performance Ranking Selection Method. The solution of multitarget optimization problem is not unique; it is generally a solution set, so the performance evaluation of candidate solutions is particularly critical. There are currently three typical traditional evaluation methods for the solution of multiobjective optimization problems: linear weighting method, reference target method, and weighted composite method. The disadvantage of these methods is that the weight setting is highly subjective and has a great influence on the results. In this paper, the performance ranking selection method is used to avoid the subjectivity of the weight setting of the traditional evaluation method, so as to make the problem solving more reasonable.

Before selecting candidate solutions, the performance ranking selection method needs to sort all candidate solutions under a single performance indicator to obtain the optimal solution under a single indicator. On the basis of sorting all performance indicators individually, the performance ranking selection is carried out. The solution with the worst ranking is selected. If there are multiple candidate solutions with the same worst ranking, the second worst ranking is considered. If the second worst ranking is also the same, the third worst ranking is considered, and so on, until the elite solution is selected.

4.3. Solution Strategy of Mathematical Model for Optimal Access Point and Capacity of Wind Turbine. In this paper, the optimization of wind turbine capacity and location is an optimization problem with discrete control variables.

The specific solving steps are as follows.

Step 1: taking the access node and capacity of the wind turbine as variables, the solution space of the access scheme of the wind turbine to be optimized is determined. Considering that the total installed capacity of wind turbines is limited by the maximum capacity of the grid, this paper limits the total number of wind turbines in the early stage. Using $p \%$ elitist sampling method, the minimum number of samples $N_{\min }^{\prime}$ is obtained for the mathematical model proposed in this paper

Step 2: randomly generate the wind turbine access scheme. The sampling strategy of each scheme is to randomly select the control variables within their variation range. The generated wind turbine access scheme needs to judge whether the scheme is the same as the existing scheme, and if it is the same, reextract it.

Step 3: calculate the power flow of the system corresponding to different randomly generated wind turbine access schemes, check the constraints such as electrical limit, and call the wind turbine access scheme that meets the constraints as the feasible wind turbine access scheme. The nonconforming schemes are directly deleted and resampled. Finally, based on the above $N_{\min }{ }^{\prime}$ feasible wind turbine access schemes, the network loss under the corresponding scheme is calculated.

Step 4: combined with different wind turbine scenes, use formulas (21) and (22) to calculate the investment cost and operation and maintenance cost of each feasible wind turbine access scheme.

Step 5: combined with different wind turbine scenes and load scenes, the short-circuit calculation is carried out for the systems corresponding to the $N_{\min }{ }^{\prime}$ feasible wind turbine access schemes. and then the economic loss of sensitive load voltage sag corresponding to each wind turbine access scheme is calculated by using formula (29). 
Step 6: calculate the corresponding objective function value of each feasible wind turbine access scheme, select the final elite solution according to the performance ranking selection method, and output the access point and access capacity of the optimal access scheme.

In summary, the flow chart of the research on the multitarget optimal access point and capacity of wind turbines is shown in Figure 2.

\section{Example Analysis}

The wind speed data used in this paper are from the simulation of HOMER. The wind speed data includes the wind speed of 24 hours in 365 days, and the wind speed of each hour is regarded as a constant value. After classifying the trend similarity of the wind speed curve, the wind speed curve is divided into 9 categories and a total of 328 wind speed data. The trend change of part of the first type wind speed curve obtained is shown in Figure 3.

Follow the scene generation steps described in Section 2.1 to get 500 sets of wind speed scenes. Because there are too many scenes, only the normalized probabilities of some scenes under the first wind speed curve are listed. See Table 2 for details.

If the wind speed curves are divided into $m$ type, for each type of wind speed curve, it will generate 500 wind speed scenes randomly, and there will be $500 * n$ wind speed scenes. The increase of the number of scenes is helpful to the real response to the original situation and the accuracy of subsequent calculation. But, at the same time, it will also increase the amount of calculation in the subsequent calculation process, which is not enough to meet the needs of engineering. Therefore, it is necessary to delete 500 sets of wind speed scenes under each type of wind speed in order to ensure a small amount of calculation and enough wind speed scenes. The final scene deletion results should be able to correctly and reasonably reflect the actual wind speed curve changes, and the number of scenes can be guaranteed in a reasonable range.

In order to ensure the validity of clustering analysis in the process of scene deletion, the actual number of clustering based on the combination of DPC \& $K$-means is set to be $2 \sim 20$, and 19 clustering schemes are obtained. Select the number of clustering schemes according to Figure 4 . The specific results are shown in the figure as follows.

It can be seen from Figure 4 that when the number of clusters is 2 , the indicator $\mathrm{CH}^{(+)}$of DPC \& $k$-means algorithm and $K$-means algorithm is the largest, which indicates that 2 is the optimal scene deletion result under this kind of wind speed. Moreover, when the number of clusters is 2, the indicator $\mathrm{CH}^{(+)}$obtained by DPC \& $k$-means algorithm is larger than that obtained by $K$-means algorithm. It shows that DPC \& $k$-means algorithm can cluster data better and get better results.

After the scene reduction process, we need to calculate the corresponding scene probability. The scene probability after scene reduction under the first wind speed curve is listed in Table 3.

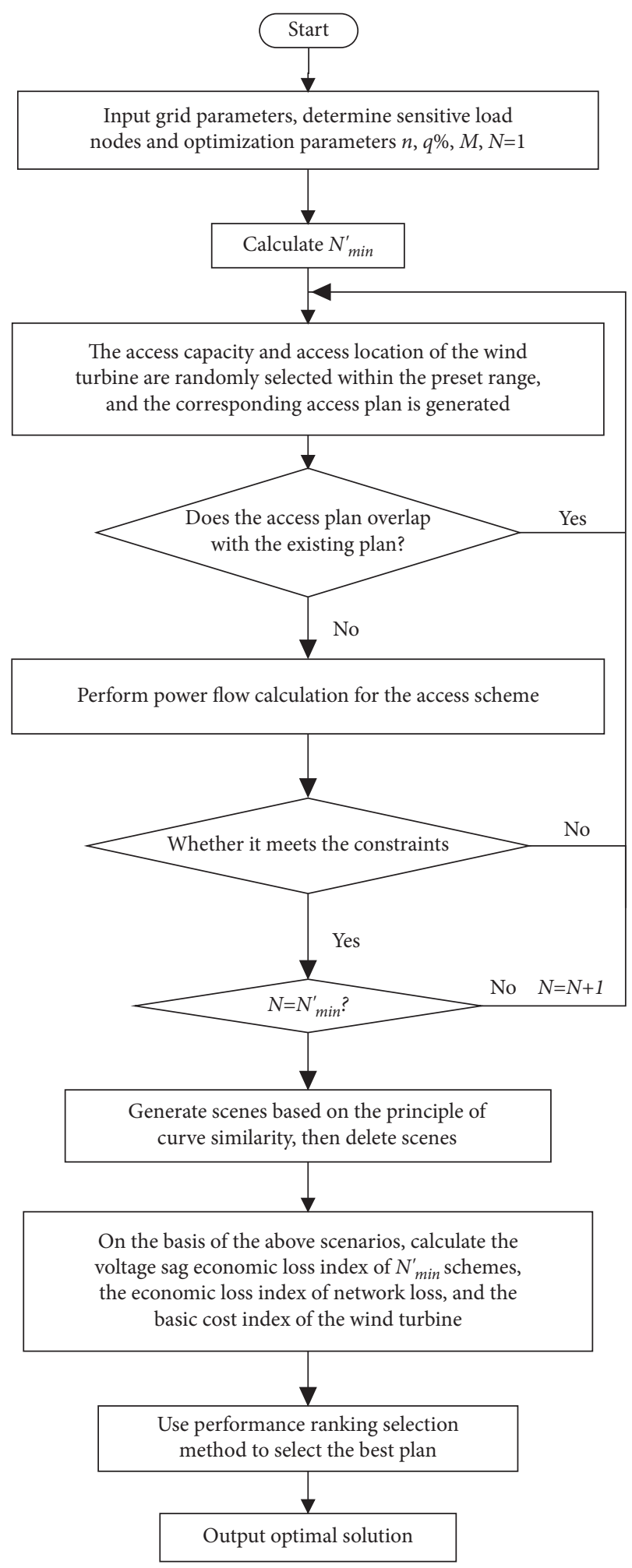

Figure 2: Flow chart of the research.

The optimization of optimal access point and capacity of wind turbine based on IEEE 33-bus system consists of 33 nodes, 32 branches, and 5 tie switch branches and operates in radial network. The five dashed lines in Figure 5 are the branches of network interconnection switch, and the 


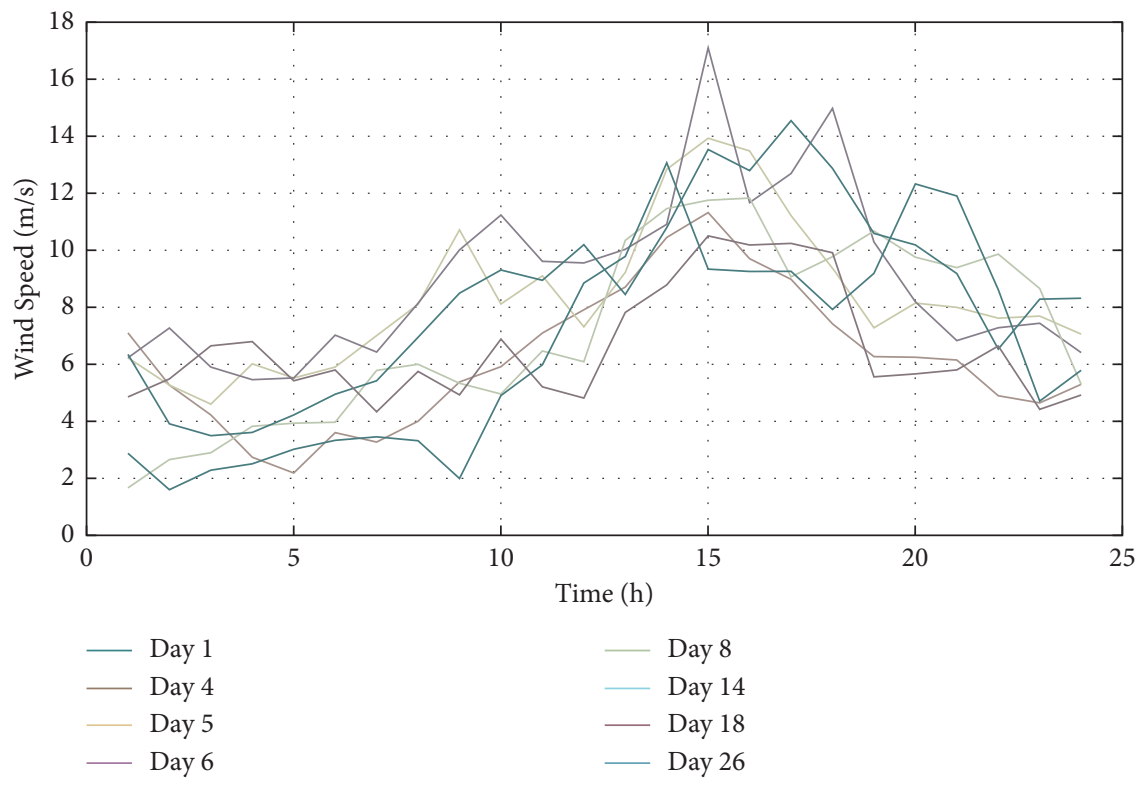

FIgURE 3: Wind speed curve trend of a certain type.

TABLE 2: Standardized probability of some scenarios under the first type wind speed curve.

\begin{tabular}{|c|c|c|c|c|c|c|c|c|}
\hline Scene 1 & Scene 2 & Scene 3 & Scene 4 & Scene 5 & Scene 6 & Scene 7 & Scene 8 & Scene 9 \\
\hline $8.78 E-05$ & $3.53 E-04$ & $3.08 E-04$ & $8.59 E-05$ & $1.80 E-03$ & $1.80 E-04$ & $2.82 E-04$ & $1.20 E-03$ & $1.70 E-04$ \\
\hline Scene 10 & Scene 11 & Scene 12 & Scene 13 & Scene 14 & Scene 15 & Scene 16 & Scene 17 & Scene 18 \\
\hline $1.50 E-03$ & $1.63 E-02$ & $1.01 E-02$ & $1.94 E-04$ & $1.55 E-04$ & $7.33 E-04$ & $8.34 E-04$ & $1.18 E-05$ & $3.08 E-04$ \\
\hline
\end{tabular}

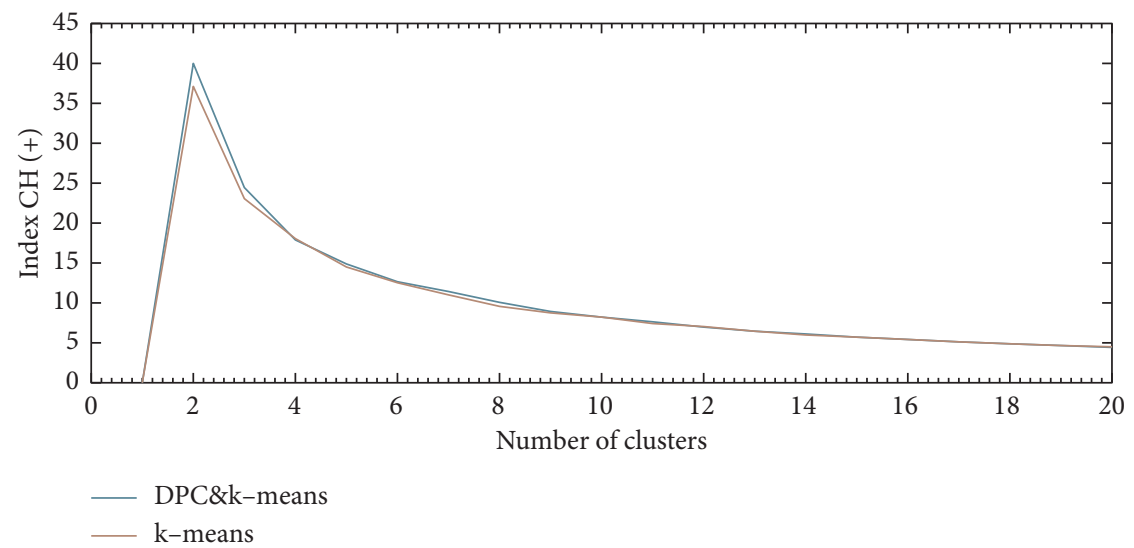

FIgURE 4: Indicator change chart under different clustering conditions.

TABLE 3: Scene probability after scene reduction under the first type wind speed curve.

\begin{tabular}{lc}
\hline Scene 1 & Scene 2 \\
\hline 0.5223 & 0.4777 \\
\hline
\end{tabular}

switches on them are normally open. The distribution system structure and line parameters in this example are from [25].

The inverter wind turbine is used for simulation verification. The inverter wind turbine adopts PV control, and the power factor is about 0.9 . There are $10,11,12,13,14,15$, $16,17,18,19$, and 20 buses that can be accessed to the wind turbine, but only 4 buses are allowed to access the wind turbine. The rated capacity of a single wind turbine is $100 \mathrm{~kW}$. The installation cost of a single wind turbine is 100,000 yuan (CNY), and the hourly operation and maintenance cost of the equipment are 400 yuan. The access point of general load is 16, and the general load has time sequence, and the data is automatically generated by the HOMER. Sensitive load nodes are 11 and 25, and the capacity of sensitive load does not change with time. The average economic loss caused by a single voltage sag event of sensitive load on bus 11 and bus 25 is 100,000 yuan (CNY) and 300,000 yuan (CNY), respectively. The economic cost of unit network loss is 1,000 yuan. The fault probabilities of single- 


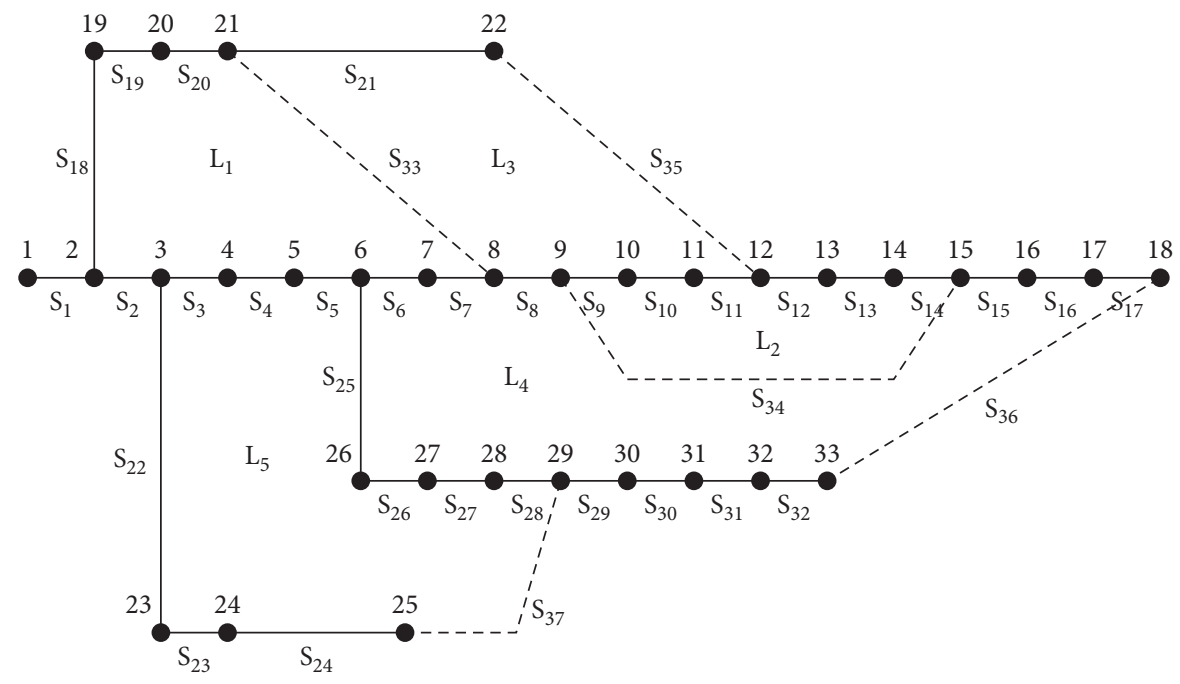

FIGURE 5: IEEE 33-bus system.

TABLE 4: Distribution of comparison and ranking of performance indicators corresponding to different solutions.

\begin{tabular}{|c|c|c|c|c|c|c|c|}
\hline Objective scheme & Rank by $f_{1}$ & Rank by $f_{2}$ & Rank by $f_{\text {sen }}$ & Worst ranking & Second worst ranking & Third worst ranking & Elite solution \\
\hline 90 & 351 & 76 & 83 & 351 & 83 & 76 & \\
\hline 91 & 89 & 439 & 84 & 439 & 89 & 84 & \\
\hline 92 & 219 & 199 & 85 & 219 & 199 & 85 & \\
\hline 93 & 323 & 129 & 86 & 323 & 129 & 86 & \\
\hline 94 & 110 & 275 & 87 & 275 & 110 & 87 & \\
\hline 120 & 220 & 5 & 113 & 220 & 113 & 5 & \\
\hline 175 & 6 & 1 & 453 & 453 & 6 & 1 & \\
\hline 95 & 66 & 68 & 88 & 88 & 68 & 66 & $\sqrt{ }$ \\
\hline 96 & 2 & 346 & 89 & 346 & 89 & 2 & \\
\hline 100 & 90 & 356 & 93 & 356 & 93 & 90 & \\
\hline 101 & 1 & 459 & 94 & 459 & 94 & 1 & \\
\hline 7 & 24 & 440 & 1 & 440 & 24 & 1 & \\
\hline 9 & 345 & 207 & 3 & 345 & 207 & 3 & \\
\hline 30 & 3 & 448 & 24 & 448 & 24 & 3 & \\
\hline 17 & 346 & 158 & 11 & 346 & 158 & 11 & \\
\hline 18 & 40 & 447 & 12 & 447 & 40 & 12 & \\
\hline 19 & 62 & 420 & 13 & 420 & 62 & 13 & \\
\hline 36 & 256 & 353 & 30 & 353 & 256 & 30 & \\
\hline 37 & 215 & 218 & 31 & 218 & 215 & 31 & \\
\hline 49 & 63 & 415 & 43 & 415 & 63 & 43 & \\
\hline
\end{tabular}

phase to ground fault, two-phase to phase fault, three-phase to phase fault, and two-phase to ground fault are $0.83,0.08$, 0.05 , and 0.04 , respectively.

According to the number of wind turbines and the change of access points, different access schemes of wind turbines are constructed. Considering that the access capacity of wind turbine is limited by the total load capacity, the number of single bus accessed to wind turbine is 8 . Therefore, the number of wind turbines that can be accessed to each bus is between 0 and 8 , and the value is randomly selected by Step 1. Finally, the total number of wind turbine access schemes (the total number of individuals in the solution space) can be calculated as $M=(10 \times 9)^{4}$, and the minimum number of samples $N_{\min }^{\prime}=459$.
There are many data about the comparison and ranking of the solutions obtained under different indicators, and only 20 groups of solutions are extracted. See Table 3 for details.

It can be seen from Table 4 that the candidate solutions are sorted in descending order and the corresponding performance indicators of different solution schemes are ranked in the corresponding indicator population. Taking the No.90 scheme as an example, the performance indicator $f_{1}$ is ranked 351 in descending order, the performance indicator $f_{2}$ is ranked 76 in descending order, and the performance indicator $f_{\text {sen }}$ is ranked 83 in descending order. On the basis of the above, through the performance ranking selection, the 95th scheme is the final elite solution, and the corresponding access capacity and access point of wind 
turbine are as follows: access node 15, 0.9 MW; access node 22, 0.7 MW; access node 23, 0.5 MW; access node 27, $0.3 \mathrm{MW}$.

\section{Conclusions}

The access of wind turbines to the distribution network has a mitigation effect on voltage sag. This paper considers the time sequence characteristics of wind power output, and, from the perspective of alleviating voltage sags, an optimization algorithm for the selection of the access point and capacity of wind turbines based on voltage sags is proposed. Several detailed conclusions are obtained:

(1) The multiscene analysis method is used to simulate the uncertainty of wind turbine output; the scenes are reasonably generated and deleted. It converts uncertain factors into deterministic factors that are easy to be obtained and calculated and solves practical problems on the basis of deterministic factors. This method effectively avoids mathematical modelling of high-complexity random problems and reduces the difficulty of solving problems.

(2) The evaluation indicator of the voltage sag depth of the bus and economic loss evaluation indicator for sensitive load voltage sag are proposed. These indicators can evaluate the mitigation degree of wind turbine access to voltage sag caused by fault. These indicators provide help and basis for the reasonable selection of the access point and capacity of wind turbines.

(3) A multiobjective mathematical model for the selection of the access point and capacity of wind turbines is proposed in this paper. This model considers the traditional basic cost of wind turbine and the economic loss of network losses and also considers the voltage sag problem that has the most complaints in power quality. It provides a new idea for the reasonable selection of the access point and capacity of wind turbines.

(4) The elite sampling algorithm is used to solve the multiobjective mathematical model proposed in this paper. It can obtain a more high-precision solution based on the reasonable solution space and avoid the situation of nonconvergence. This algorithm has the characteristics of simplicity and easiness to understand, small amount of calculation, and high applicability, and it is suitable for solving practical engineering problems.

(5) The IEEE 33-bus system example is used to verify the optimization algorithm proposed in this paper. The results show that the wind turbine accessing scheme determined by the algorithm can not only reduce the network loss but also alleviate the voltage sag.

\section{Data Availability}

The data used to support the findings of this study are available from the corresponding author upon request.

\section{Conflicts of Interest}

The authors declare that they have no conflicts of interest.

\section{Acknowledgments}

This paper was supported by the National Natural Science Foundation of China under Grant 51777166.

\section{References}

[1] A. S. Morteza, S. Sakineh, O. Cagil, and A. Kalam, "Developing a mathematical model for wind power plant siting and sizing in distribution networks," Energies, vol. 13, no. 13, 2020.

[2] C. Peng, R. Yu, and H. Sun, "Multi-objective planning of distributed power generation based on $K$-means clustering multi-scenario time sequence analysis," Power Automation Equipment, vol. 35, no. 10, pp. 58-65, 2015.

[3] Q. Yuan, K. Zhou, and J. Yao, "A new measure of wind power variability with implications for the optimal sizing of standalone wind power systems," Renewable Energy, vol. 150, pp. 538-549, 2020.

[4] D. Rama Prabha, T. Jayabarathi, R. Umamageswari, and S. Saranya, "Optimal location and sizing of DG unit using intelligent water drop algorithm," Sustainable Energy Technologies and Assessments, vol. 11, pp. 106-113, 2015.

[5] J. J. Jamian, M. N. Muhtazaruddin, and M. A. Baharudin, "Penalty factor-based optimization algorithm for distributed generation sizing in distribution network," Turkish Journal of Electrical Engineering and Computer Sciences, vol. 24, no. 3, pp. 1815-1825, 2016.

[6] L. Luo, W. Gu, X.-P. Zhang et al., "Optimal siting and sizing of distributed generation in distribution systems with PV solar farm utilized as STATCOM (PV-STATCOM)," Applied Energy, vol. 210, pp. 1092-1100, 2018.

[7] S. Zhao, S. Xun, Y. Ma, and L. Dong, "Renewable energy widearea complementary planning method based on complex adaptive system theory," Power Grid Technology, vol. 44, no. 10 , pp. $3671-3681,2020$.

[8] S. Zeynali, N. Rostami, M. R. Feyzi, and B. MohammadiIvatloo, "Multi-objective optimal planning of wind distributed generation considering uncertainty and different penetration level of plug-in electric vehicles," Sustainable Cities and Society, vol. 62, Article ID 102401, 2020.

[9] N. Z. Mohd Ali, I. Musirin, Z. Khalidin, and M. R. Ahmad, "Distributed generation sizing and placement using computational intelligence," in Proceedings of the IEEE International Power Engineering and Optimization Conference (PEOCO2012), pp. 527-532, Melaka, Malaysia, June 2012.

[10] S. Mahajan and S. Vadhera, "Optimal sizing and deploying of distributed generation unit using a modified multi-objective Particle Swarm Optimization," in Proceedings of the IEEE 6th International Conference on Power Systems (ICPS), pp. 1-6, New Delhi, India, 2016. 
[11] X. Yang, J. Yang, and J. Deng, "Location and capacity of distributed power sources based on optics optimization algorithm," Power Supply, vol. 35, no. 11, pp. 66-71, 2018.

[12] G. Lu, T. Huan, and H. Liao, "Location and capacity of distributed power sources based on improved searching algorithm of beef whisk," Electrical Measurement And Instrumentation, vol. 56, no. 718, pp. 14-20, 2019.

[13] J. Yang, R. Zhang, Q. Sun, and H. Zhang, "Optimal wind turbines micrositing in onshore wind farms using fuzzy genetic algorithm," Mathematical Problems in Engineering, vol. 2015, Article ID 324203, 9 pages, 2015.

[14] J. Aghaei, T. Niknam, R. Azizipanah-Abarghooee, and J. M. Arroyo, "Scenario-based dynamic economic emission dispatch considering load and wind power uncertainties," International Journal of Electrical Power \& Energy Systems, vol. 47, no. MAY, pp. 351-367, 2013.

[15] Y. Zhou, Research on Integral Displacement Algorithm of Acceleration Test and its Application, ChongQing University, Chong Qing, China, 2013.

[16] Z. Michalewicz, Genetic Algorithms + Data Structures = Evolution Programs, Springer, New York, NY, USA, 3rd ed edition, 1996.

[17] A. Rodriguez and A. Laio, "Clustering by fast search and find of density peaks," Science, vol. 344, no. 6191, pp. 1492-1496, 2014.

[18] S. Ding and Q. Tian, "Ball-Tree optimized density peak clustering algorithm," Computer Engineering and Applications, vol. 1-9, 2021.

[19] R. B. Calinski and J. Harabasz, "A dendrite method for cluster analysis," Communications in Statistics, vol. 3, no. 1, pp. 1-27, 1974.

[20] E. Clarke, Circuit Analysis of AC Power Systems, Wiley, New York, NY, USA, 1943.

[21] L. Ma, D. Tian, T. Liu, S. Gao, and P. Dong, "An evaluation method for bus and grid structure based on voltage sags/swells using voltage ellipse parameters," IEEE ACCESS, vol. 9, Article ID 101959, 2021.

[22] V. Ignatova, P. Granjon, and S. Bacha, "Space vector method for voltage dips and swells analysis," IEEE Transactions on Power Delivery, vol. 24, no. 4, pp. 2054-2061, 2009.

[23] C. Becker, W. Braun, K. Carrick et al., "Proposed chapter 9 for predicting voltage sags(dips) in revision to IEEE Std.493 the gold book," IEEE Transactions on Industry Applications, vol. 30, no. 3, pp. 805-821, 1994.

[24] J. Liu and F. Qian, Engineering Analysis Methods-Simplified Analysis Methods for Engineering Applications, China Water Conservancy and Hydropower Press, Beijing, China, 2017.

[25] L. Ma, T. Liu, and P. Dong, "The sizing and siting of DistributedGeneration for mitigating voltage sags," in Proceedings of the 6th International Conference on Energy Materials and Environmental Engineering, Tianjin, China, 2020. 